\title{
A theoretical modeling framework for motile and colonial harmful algae
}

\author{
Jackie Taylor ${ }^{1}$, M. Carme Calderer ${ }^{1}$, Miki Hondzo $^{1}$, and Vaughan Voller ${ }^{1}$ \\ ${ }^{1}$ University of Minnesota Twin Cities
}

December 8, 2021

\begin{abstract}
1. Harmful algal blooms are increasing in both severity and frequency across the globe. Many bloom-forming species are capable of vertical motility and colony formation. The cyanobacterium Microcystis aeruginosa is a common example of such a species, yet current models poorly predict vertical distributions of $M$. aeruginosa. 2. To couple the hydrodynamics, buoyancy, and the colony dynamics of Microcystis, we present a system of one-dimensional advection-diffusion-aggregation equations with Smoluchowski aggregation terms. 3. Results indicate Smoluchowski aggregation accurately describes the colony dynamics of $M$. aeruginosa. Further, transport dynamics are strongly dependent on colony size, and aggregation processes are highly sensitive to algal concentration and wind-induced mixing. Both of these findings have direct consequences to harmful algal bloom formation. 4. While the theoretical framework outlined in this manuscript was derived for M. aeruginosa, both motility and colony formation are common among bloom-forming algae. As such, this coupling of vertical transport and colony dynamics is a useful step for improving forecasts of surface harmful algal blooms.
\end{abstract}

\section{Hosted file}

ADA_MEE.pdf available at https://authorea.com/users/450114/articles/548508-a-theoreticalmodeling-framework-for-motile-and-colonial-harmful-algae 